\title{
Proyecto de intervención para la capacitación virtual de profesores de educación superior en diseño instruccional y recursos educativos digitales
}

\author{
Intervention project for virtual training of higher education teachers in \\ instructional design and digital educational resources
}

Projeto de intervenção para a formação virtual de professores do ensino superior em design instrucional e recursos educacionais digitais

\author{
Luis Alejandro Gazca Herrera \\ Universidad Veracruzana, México \\ Igazca@uv.mx \\ https://orcid.org/0000-0001-7637-2909
}

\section{Resumen}

La pandemia generada por la covid-19 ha tenido un impacto negativo en los ámbitos económicos, sociales y educativos. La contingencia ha obligado a que más personas, sin importar su profesión o actividad, recurran a plataformas digitales para prepararse en el aspecto profesional y académico. Este artículo, por tanto, tiene como objetivo presentar los resultados de un trabajo de investigación evaluativa llevado a cabo para medir la implementación del proyecto de intervención sobre la capacitación de profesores de educación superior en el diseño instruccional y en la producción de recursos educativos digitales. Para evaluar el proyecto, se elaboró un instrumento, el cual se aplicó en línea luego de la capacitación para identificar los resultados descriptivos e inferenciales. Para la obtención de los datos y su análisis respectivo, se aplicó una metodología de corte mixto que consideró diferentes momentos, actores e instrumentos. En concreto, se aplicó un enfoque cuantitativo. La investigación se llevó a cabo en la Universidad Veracruzana, específicamente con la participación de profesores del área académica técnica. Los resultados señalan que el proyecto de intervención tuvo resultados favorables en cuanto al diseño de cursos en línea bajo una metodología de diseño instruccional con el uso de recursos 


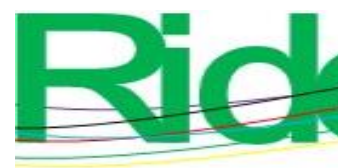

Revista Iberoamericana para la Investigación y el Desarrollo Educativo ISSN $2007-7467$

educativos digitales, pues se incrementó la acreditación en las materias de la modalidad mixta; sin embargo, es necesario realizar otro estudio que contraponga las percepciones de los profesores y de los estudiantes sobre la mejora en los procesos de enseñanza-aprendizaje.

Palabras clave: capacitación, diseño instruccional, evaluación, proyecto de intervención, recursos educativos digitales.

\section{Abstract}

The COVID-19 pandemic has had a negative impact on the economic, social and educational spheres, the contingency has led more people, regardless of their profession or activity, to turn to digital platforms to prepare in the professional and academic sphere. The present research aims to show the results of an evaluative research work, carried out to measure the implementation of the intervention project on the training of higher education teachers in instructional design and in the production of digital educational resources. To evaluate the project, an instrument was developed that was applied online, after the training, to clearly identify the descriptive and inferential results. To recover the data and its corresponding analysis, a mixed cut methodology was used that considered different moments, actors and instruments. This document presents the work carried out using the quantitative approach, highlighting the results obtained in the intervention groups. The research was carried out at the Universidad Veracruzana, specifically with the participation of professors from the technical academic area. The results indicate that the intervention project had favorable results, in terms of the design of online courses under an instructional design methodology with the use of digital educational resources, increasing the accreditation of the subjects under the mixed modality, however, it is It is necessary to carry out another study that contrasts the perceptions of teachers and students about the improvement in the teachinglearning processes.

Keywords: capacitation, instructional design, evaluation, intervention project, digital educational resources. 


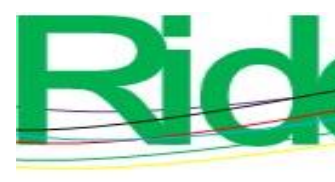

Revista Iberoamericana para la Investigación y el Desarrollo Educativo ISSN $2007-7467$

\section{Resumo}

A pandemia gerada por covid-19 teve um impacto negativo nas esferas econômica, social e educacional. A contingência tem forçado mais pessoas, independentemente de sua profissão ou atividade, a recorrer às plataformas digitais para se preparar profissional e academicamente. Este artigo, portanto, tem como objetivo apresentar os resultados de uma pesquisa avaliativa realizada para medir a implementação do projeto de intervenção na formação de professores do ensino superior em design instrucional e na produção de recursos educacionais digitais. Para avaliação do projeto, foi desenvolvido um instrumento, o qual foi aplicado online após o treinamento para identificar os resultados descritivos e inferenciais. Para a obtenção dos dados e respetiva análise, foi aplicada uma metodologia de recortes mistos que considerou diferentes momentos, atores e instrumentos. Especificamente, uma abordagem quantitativa foi aplicada. A pesquisa foi desenvolvida na Universidade Veracruzana, especificamente com a participação de docentes da área acadêmica técnica. Os resultados indicam que o projeto de intervenção teve resultados favoráveis ao nível da concepção de cursos online sob uma metodologia de design instrucional com a utilização de recursos educacionais digitais, uma vez que foi aumentada a acreditação nas disciplinas da modalidade mista; No entanto, é necessário realizar outro estudo que compare as percepções de professores e alunos sobre a melhoria dos processos de ensino-aprendizagem.

Palavras-chave: treinamento, design instrucional, avaliação, projeto de intervenção, recursos educacionais digitais.

Fecha Recepción: Abril 2021

Fecha Aceptación: Octubre 2021

\section{Introducción}

La pandemia que actualmente vivimos debido a la covid-19 ha traído cambios significativos en todos los ámbitos, incluido el educativo. Por eso, hubo la necesidad de pasar abruptamente de una educación presencial a una en línea, lo cual provocó que se vieran afectados los procesos de enseñanza y aprendizaje no solo porque muchas instituciones no contaban con la infraestructura tecnológica necesaria, sino principalmente porque la mayoría de los docentes carecían de competencias digitales para afrontar el nuevo escenario educativo.

En efecto, según un estudio desarrollado en la Universidad Veracruzana (UV), se pudo conocer que solo $43 \%$ de los estudiantes considera que los profesores tienen las 


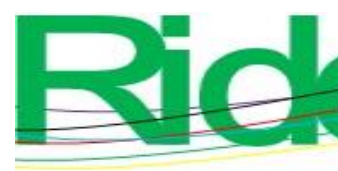

Revista Iberoamericana para la
Investigación y el Desarrollo Educativo
ISSN $2007-7467$

habilidades necesarias para impartir las clases bajo la modalidad virtual, aunque estas cifras contrastan con los datos recabados por Gazca (2020), quien señala que 79\% de los profesores creen que tienen las competencias necesarias para desarrollar con efectividad en la modalidad virtual los procesos de enseñanza y aprendizaje. Estos resultados son fundamento para llevar a cabo un proyecto de intervención de capacitación en el diseño instruccional y en la producción de recursos educativos digitales con base en la plataforma tecnológica de la UV.

\section{Marco conceptual}

\section{El diseño instruccional}

Capacitar a los profesores en cooperación con las TIC es vital para la mejora continua; no obstante, lo complicado es que para trabajar en medios virtuales se debe mejorar la planeación didáctica que se sustenta en una metodología denominada diseño instruccional, la cual se encarga de la selección apropiada de técnicas para la enseñanza-aprendizaje, recursos tecnológicos, así como de la identificación de medios educativos y medición del manejo (Branch y Kopcha 2014; Domínguez et al., 2018; Moreno et al., 2014).

El diseño instruccional, por tanto, es un proceso sistemático, fundamentado, planificado y estructurado que permite al alumno apropiarse de los conocimientos de determinada materia por medio de instrucciones exactas distribuidas en cuatro entornos: las ocupaciones preliminares, los ejercicios, la actividad integradora y un caso integrador, por lo que se convierte en un mediador del aprendizaje con propiedades autodidactas (Brambila et al., 2006). En concreto, las etapas del modelo instruccional son las siguientes: análisis, diseño, desarrollo, implementación y evaluación (ADDIE), las cuales se relacionan entre sí (Luna, Ayala y Rosas, 2021).

Por eso, de acuerdo con Belloch (2013), el modelo instruccional también es múltiple porque se ajusta a las necesidades del profesor, lo que permite su adecuación para el logro de los objetivos trazados. Igualmente, otro de sus aspectos relevantes es la manera en que se desarrollan las indicaciones, pues brindan pautas específicas para realizar secuencias didácticas, lo que resulta en un acercamiento de enseñanza-aprendizaje de forma sincrónica (en tiempo real) como asincrónica. No obstante, una vez diseñada la instrucción, debe ser probada, evaluada y revisada para que atienda de manera eficaz las necesidades particulares del curso desarrollado (Candia, 2016). 


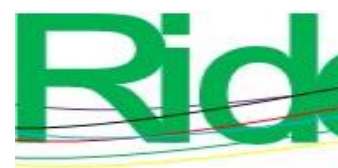
Revista Iberoamericana para la
Investigación y el Desarrollo Educativo
ISSN $2007-7467$

\section{Los recursos digitales}

Con base en diversos estudios, se puede asegurar que los recursos tecnológicos por sí mismos no pueden resolver todos los inconvenientes que presentan las instituciones educativas (López et al., 2013). De hecho, si no son bien aprovechados pueden crear en los alumnos apatía y, a largo plazo, deserción escolar. Por eso, se puede afirmar que el dominio de las TIC por parte de los docentes es una variable que resulta muy útil para fomentar el aprendizaje de los estudiantes.

Al respecto, la Organización de las Naciones Unidas para la Educación, Ciencia y Cultura señala algunos principios básicos que se deben tomar en cuenta en todos los centros educativos:

- Debería integrarse la tecnología a todo el programa de formación docente.

- Los futuros maestros tienen que formarse y experimentar en ámbitos educativos que hagan un uso innovador de la tecnología (López et al., 2013; Unesco, 2004).

Ahora bien, cabe resaltar que los elementos tecnológicos deben ser evaluados una vez implementados para detectar áreas que puedan ser mejoradas (Aguaded y Muñoz, 2012; Margalef y García, 2016). Por ello, para que este tipo de herramientas tecnológicas puedan ser catalogadas como educativas deben fomentar el aprendizaje de contenidos conceptuales y procedimentales, así como contribuir a la educación integral del individuo (Zapata, 2012).

Lógicamente, en el mercado existen diversas aplicaciones que permiten crear recursos educativos digitales sin necesidad de ser un experto en la materia. Por ejemplo, para diseñar videotutoriales se puede utilizar Open Broadcaster Software (OBS), el cual permite grabar videos y hacer transmisiones en vivo. Otra de las aplicaciones es Videopad, herramienta de edición de videos desarrollada por NCH Software (disponible para Windows, Mac, Iphone, Ipad y Android). Con VideoPad se puede conseguir un producto con una apariencia profesional en minutos. Asimismo, Audacity es un software editor y grabador de audio, de código abierto y gratuito, compatible con Windows, macOS, GNU/Linux, entre otros. Puede exportar grabaciones en muchos formatos. 


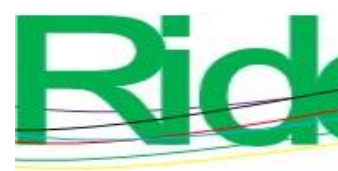

Revista Iberoamericana para la
Investigación y el Desarrollo Educativo
ISSN $2007-7467$

\section{Plataformas educativas virtuales}

Las plataformas educativas virtuales brindan la posibilidad no solo de publicar los recursos educativos digitales, sino también de promover la interacción entre todos los participantes (Andrade y Velázquez, 2014). Además, sirven para que los maestros evalúen y hagan seguimiento de los avances de los alumnos gracias a indicadores que las propias herramientas brindan (Raichman y Mirasso, A. 2018).

Estas plataformas educativas deben conformarse en un ámbito informático que cuenten con herramientas agrupadas y optimizadas para objetivos docentes. Su funcionalidad es permitir la construcción y administración de cursos enteros por internet sin que sean necesarios conocimientos profundos de programación. En otras palabras, deben ser lo más sencillas de utilizar tanto para profesores como para alumnos.

\section{Marco referencial}

El objetivo de este proyecto de intervención consta de tres partes: la primera, formalizar el modelo mixto de educación en línea; la segunda, ofrecer claridad con respecto a los procesos de capacitación que se tienen y se llevan a cabo actualmente, y, por último, detallar de manera extensa todos los parámetros posibles en cuanto a la precisión y claridad que se tenga para llevar a cabo la educación en línea. Al final de todo el proceso, se tendrá suficiente información para saber exactamente qué aspectos podrán ser modificados a través de la retroalimentación de los involucrados.

Asimismo, teniendo como fundamento el documento emitido por la Secretaría Académica de la Universidad Veracruzana sobre las Disposiciones Generales para el Trabajo Académico (2020) (propuesto en el periodo escolar septiembre 2020-febrero 2021), se estableció - gracias al trabajo a distancia de emergencia que se llevó a cabo por parte de los profesores con los estudiantes durante la contingencia sanitaria ante la pandemia por la covid19 durante periodo escolar febrero 2020-julio 2020 - la existencia de los siguientes tres escenarios:

- Se tuvieron las condiciones necesarias para avanzar en los contenidos de las asignaturas conforme a lo programado durante el periodo de confinamiento.

- Existieron condiciones favorables para el reforzamiento de contenidos y avanzar en un cierto porcentaje del programa de estudios. 


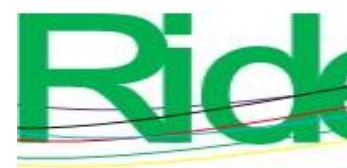

Revista Iberoamericana para la Investigación y el Desarrollo Educativo ISSN 2007 - 7467

- No existieron condiciones para el trabajo académico remoto, lo que implicó dificultades para el avance en los saberes de las asignaturas.

Por lo expuesto con anterioridad, se determinó que el periodo escolar septiembre 2020-febrero 2021, independientemente de las condiciones del semáforo de riesgo, representó una posibilidad para que los profesores fortalecieran las estrategias que probaron efectividad de su trabajo en línea. Por eso, el documento sobre Disposiciones Generales para el Trabajo Académico (2020) estableció las actividades bajo la modalidad mixta, la cual es una forma de trabajo pedagógica que considera la impartición de las asignaturas con actividades combinadas, generalmente de tipo presencial y no presencial, sobre una base de interacción favorable entre académicos y alumnos.

Para ello, el proceso de enseñanza-aprendizaje, lógicamente, requiere un uso activo de la tecnología para fijar encuentros académicos virtuales (sincrónicos y/o asincrónicos) que permitan la orientación y la entrega de actividades. En consecuencia, tendremos un ambiente de aprendizaje guiado, aunque al mismo tiempo fomentaremos la autonomía.

Asimismo, el documento antes señalado indica que los docentes deben usar plataformas educativas (preferentemente la plataforma institucional Eminus), lo cual, por supuesto, demanda conocimientos sobre estrategias en entornos virtuales de aprendizaje. Sin embargo, es claro que no todos los profesores cuentan con esos requerimientos para impartir clases en la modalidad mixta. Por ende, en coordinación con la Dirección del Área Académica Técnica de la UV, se tomó la decisión de capacitar a un número determinado de profesores de las cinco regiones donde tiene presencia la UV en el estado de Veracruz para que cumplan con éxito el mencionado propósito.

\section{Objetivo de la investigación}

El objetivo de la investigación fue evaluar, con base en el proyecto de intervención, si los académicos que habían participado en la capacitación desarrollaron competencias a partir del conocimiento de las teorías de aprendizaje, el dominio de las etapas del diseño instruccional y el uso de recursos tecnológicos que les permitieran estar formados en el uso de plataformas educativas (Eminus), habilitados tecnológicamente en los saberes tecnológicos propios de la docencia aplicando estrategias en el diseño instruccional para la elaboración de entornos virtuales de aprendizaje de las experiencias educativas bajo la modalidad mixta, y si existió una mejora en los procesos de enseñanza de los estudiantes. 


\section{Revista Iberoamericana para la Investigación y el Desarrollo Educativo ISSN $2007-7467$}

\section{Método}

Para la ejecución de este proyecto de intervención se realizó una investigación de tipo no experimental, exploratoria, descriptiva y cuantitativa. Para ello, se llevó a cabo la evaluación del impacto de la capacitación de los profesores y de la percepción que tuvieron sobre el aprendizaje de los estudiantes. Asimismo, se sustentó en los principios de la investigación descriptiva, mediante la revisión de conceptos en las principales revistas internacionales sobre los elementos del diseño instruccional, así como la elaboración de recursos educativos digitales y el manejo de plataformas tecnológicas virtuales.

\section{Población y muestreo}

Los sujetos de estudio fueron aquellos profesores pertenecientes al nivel superior de la Dirección del Área Académica Técnica (DGAAT) de la UV. De acuerdo con la numerología de la DGAAT, la planta docente en las cinco regiones que cubre el estado de Veracruz se conforma como se indica en la tabla 1.

Tabla 1. Profesores adscritos a programas educativos de la DGAAT

\begin{tabular}{|l|l|l|l|}
\hline Grado académico & Hombres & Mujeres & Total \\
\hline Doctorado & 249 & 179 & 428 \\
\hline Maestría & 99 & 89 & 188 \\
\hline Especialización & 14 & 6 & 20 \\
\hline Licenciatura & 57 & 35 & 92 \\
\hline Total & 419 & 309 & 728 \\
\hline
\end{tabular}

Fuente: Dirección General Académica del Área Técnica UV (2021)

Para determinar el tamaño de la muestra se utilizó la fórmula para el cálculo de población finita a conveniencia con la siguiente fórmula:

En donde

$$
n=\frac{N * Z^{2} * p * q}{e^{2} *(N-1)+Z^{2} * p * q}
$$

$\mathrm{Z}=$ Nivel de confianza $93 \%$, valor de tablas (1.81)

$\mathrm{p}=$ Probabilidad de que ocurra el evento $50 \%=.5$

$\mathrm{q}=(1-\mathrm{p})$ Probabilidad de que no ocurra el evento $50 \%=.5$

$\mathrm{N}=$ Población de 728 profesoras(es)

e $=$ Error de estimación máximo aceptado es de $7 \%$. 


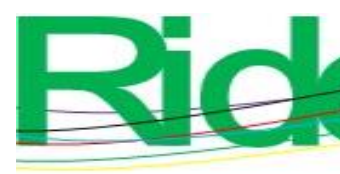

\section{Revista Iberoamericana para la Investigación y el Desarrollo Educativo ISSN 2007 - 7467}

Sustituidos los valores anteriores, obtenemos una muestra de 136 profesores que debieron tomar el curso. De acuerdo con el muestreo y su conveniencia, fueron de tiempo completo, medio tiempo o de asignatura, además del área académica técnica y perteneciente a alguna de las cinco regiones que cubre la UV en el estado de Veracruz.

La tabla 2 muestra la distribución de los profesores en los cursos y el resultado de la eficiencia terminal en cuanto al índice de aprobación del curso-taller.

Tabla 2. Profesores adscritos a programas educativos de la DGAAT

\begin{tabular}{|c|c|c|c|}
\hline Grupo & Inscritos & Aprobados & No aprobados \\
\hline 1 & 34 & 30 & 1 \\
\hline 2 & 32 & 30 & 2 \\
\hline 3 & 35 & 31 & 7 \\
\hline 4 & 35 & 25 & 10 \\
\hline Total & 136 & 116 & 20 \\
\hline \multicolumn{2}{|c|}{ Eficiencia terminal } & $85 \%$ & $15 \%$ \\
\hline
\end{tabular}

Fuente: Elaboración propia

\section{Diseño del curso de capacitación}

Para la capacitación de los profesores se elaboró un curso denominado Diseño instruccional y recursos educativos digitales, el cual fue concebido como un curso-taller en una modalidad innovadora de los procesos de enseñanza de la oferta académica. Forma parte del Programa de Formación de Académicos (ProFA) de la UV, con una duración de 45 horas (15 teóricas y 30 de práctica) y un valor de 3 créditos. La orientación del curso estuvo enfocada al diseño y producción de recursos educativos con el apoyo de aplicaciones para la edición de audios y videos que mejoran los procesos de enseñanza-aprendizaje y del desarrollo de competencias mediante entornos digitales.

Las evidencias de desempeño que debieron cumplir los inscritos al curso-taller se enfocaron a la planeación del diseño instruccional, además de utilizar los recursos tecnológicos que complementan el diseño instruccional para la aplicación de escenarios virtuales de aprendizaje.

El curso-taller contó con recursos educativos digitales para consolidar la modalidad educativa mixta, forma de trabajo pedagógico que se caracteriza por el desarrollo de experiencias educativas (materias), con interacción combinada de tipo presencial y no presencial para armonizar los procesos de enseñanza-aprendizaje con el uso de la tecnología. 
Figura 1. Formato de instrumento del diseño instruccional

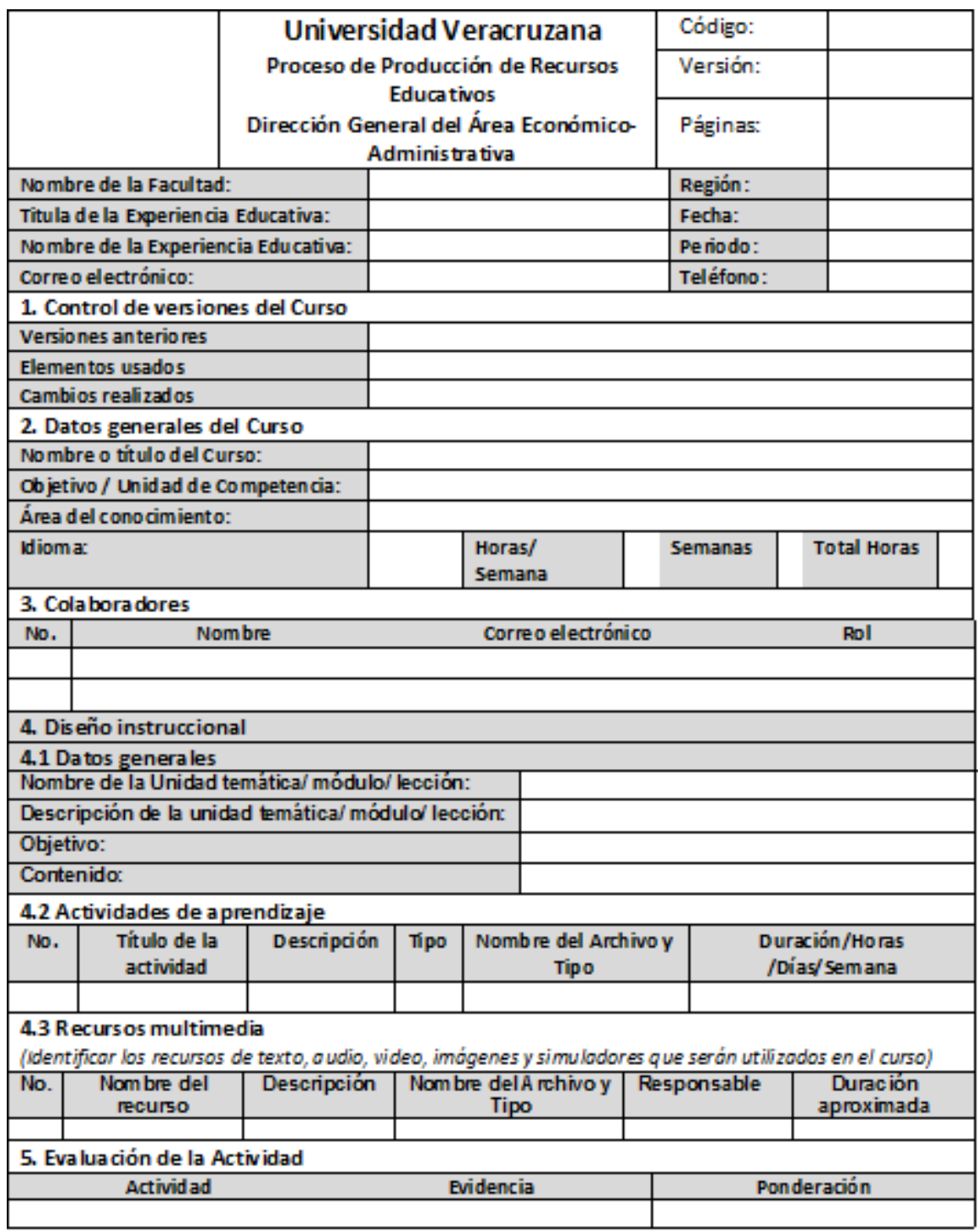

Fuente: Elaboración propia

La capacitación se efectuó en línea utilizando la plataforma virtual educativa de la UV, denominada Eminus (Sistema de Educación Distribuida de la Universidad Veracruzana). Es un sistema de administración de ambientes flexibles de aprendizaje que ayuda a presentar cursos en línea para distribuirse en internet. Este sistema posibilita la comunicación en forma sincrónica y asincrónica, y genera un ámbito completo para el aprendizaje, el cual sirve para mejorar los niveles educativos sin parámetros de tiempo o distancia. Así, cada usuario toma el control de su aprendizaje (Colunga, 2005).

Para acreditar el curso, los profesores debieron diseñar una materia en la modalidad mixta virtual. La figura 2 muestra la interfaz del curso en la plataforma Eminus. Inicialmente, se da un mensaje de bienvenida acompañado con un video que explica la operatividad del 

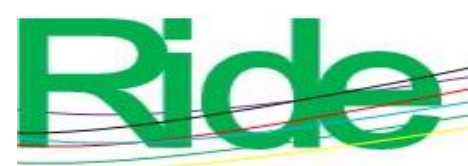

Revista Iberoamericana para la Investigación y el Desarrollo Educativo ISSN 2007 - 7467

curso. Todos los recursos educativos digitales fueron diseñados con las aplicaciones de OBS studio, Videopad y Audacity en sus versiones libres, por lo que no representó costos adicionales.

Figura 2. Interfaz del curso en la plataforma Eminus

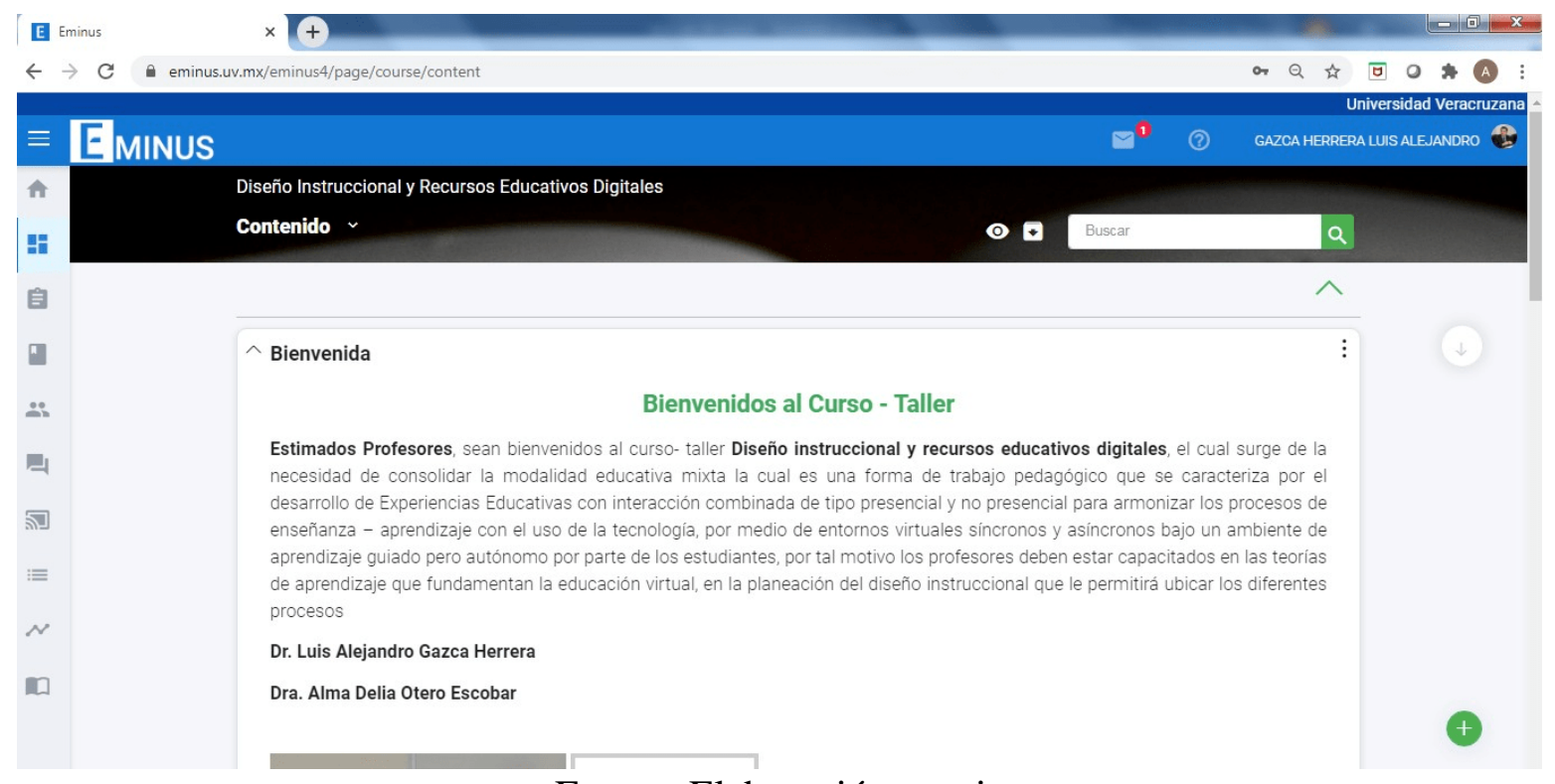

Fuente: Elaboración propia

Parte primordial del curso fue lo referente al diseño instruccional, por lo que se explicó de manera clara (con ejemplos prácticos) cómo debía ser su llenado. Se elaboraron cuatros videos que complementaron los aspectos teóricos para que los profesores pudieran elaborar su propio diseño instruccional de una de las asignaturas que imparten. La figura 3 muestra la interfaz de dicho tema. 


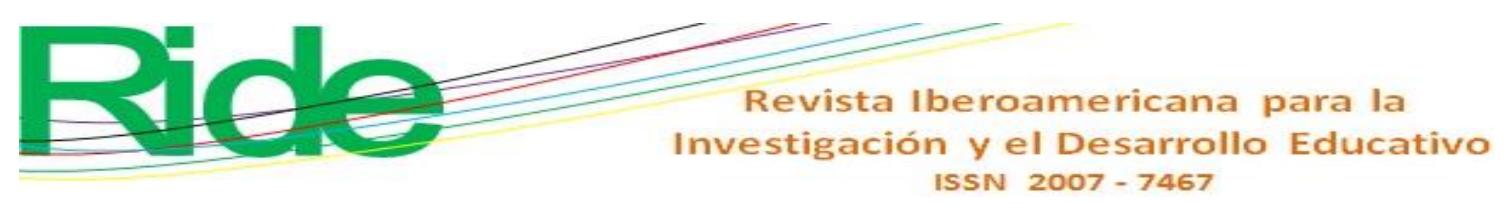

Figura 3. Interfaz del tema sobre diseño instruccional

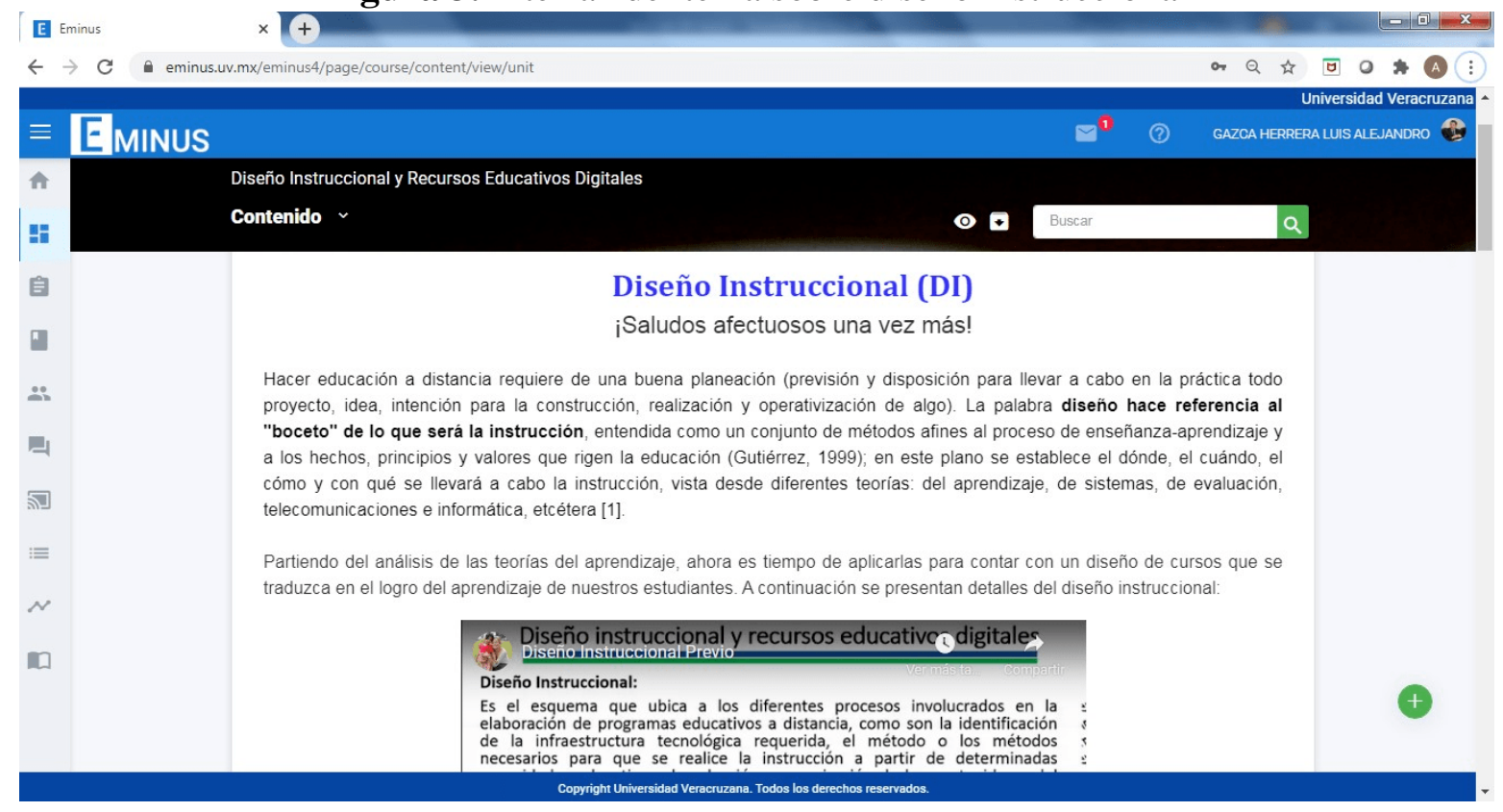

Fuente: Elaboración propia

Algo esencial del curso fue brindar las competencias necesarias para que los profesores pudieran crear sus propios recursos educativos digitales. Por ello, se consideraron tres apartados donde se crearon videotutoriales para explicar desde la instalación de las aplicaciones OBS Studio, VideoPad y Audacity hasta la forma de diseñar y editar audios y videos.

La figura 4 enseña la interfaz del apartado sobre el manejo de las aplicaciones para el diseño de recursos educativos digitales. Este conocimiento serviría para que pudieran crear un canal en YouTube, es decir, materiales distintos a los tradicionales archivos de texto. 

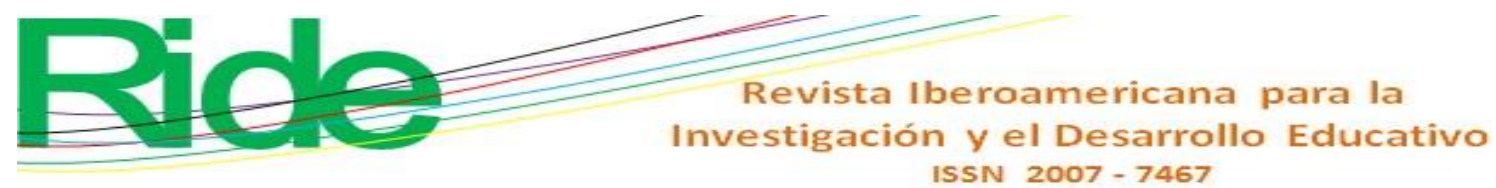

Figura 4. Interfaz del tema sobre el diseño de recursos educativos digitales

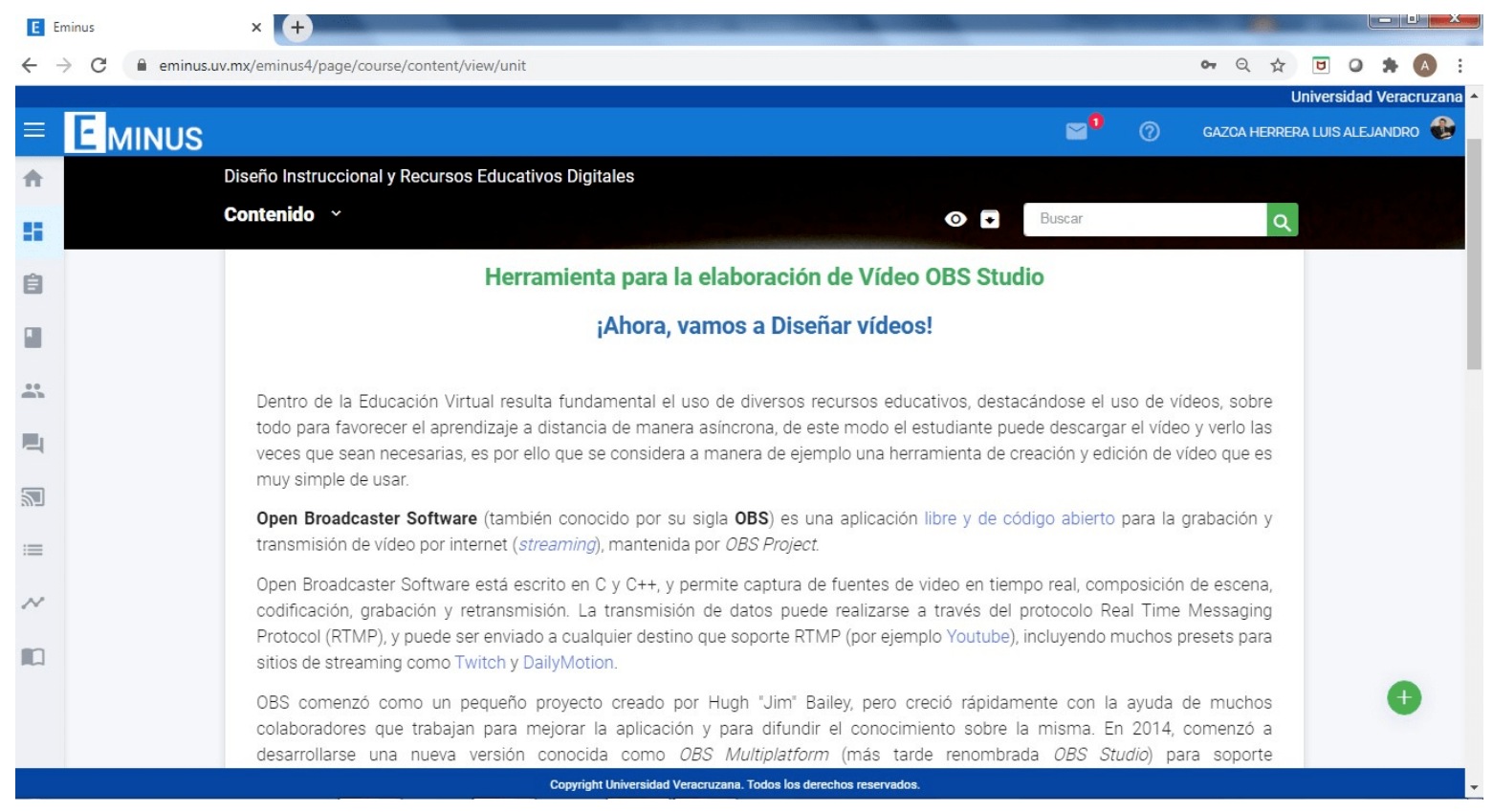

Fuente: Elaboración propia

\section{Resultados}

La evaluación es un aspecto clave en la implementación de cualquier proyecto educativo porque permite identificar variables que pueden ser mejoradas en los procesos de enseñanza y aprendizaje. Por eso, como requisito de evaluación, los participantes tuvieron que emplear la metodología de diseño instruccional para elaborar y utilizar recursos digitales en al menos una asignatura durante el periodo escolar agosto 2020-enero 2021. Por ende, se diseñó un instrumento (conformado por una escala de Likert) para medir las dimensiones de capacitación, tecnología e impacto en el aprendizaje de los estudiantes.

El instrumento quedó conformado por 15 ítems (anexo 1). La fiabilidad y la validez se analizaron con el coeficiente alfa de Cronbach, mediante el software SPSS. Las tablas 4 y 5 representan la fiabilidad de la escala del instrumento obtenida en una encuesta piloto (15 ítems) en la que participaron 20 profesores. Se obtuvo un alfa de Cronbach de .810, lo que representa un valor bueno, de acuerdo con George y Mallery (2003). 


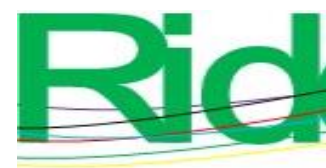

Revista Iberoamericana para la Investigación y el Desarrollo Educativo ISSN 2007 - 7467

Tabla 4. Resumen del procesamiento de los casos de las variables para profesores

\begin{tabular}{|c|l|c|c|}
\hline \multicolumn{4}{|c|}{ Resumen del procesamiento de los casos } \\
\hline \multirow{3}{*}{ Casos } & Válidos & N & $\%$ \\
\cline { 2 - 4 } & Excluidos $^{\mathrm{a}}$ & 0 & 100.0 \\
\cline { 2 - 4 } & Total & 20 & .0 \\
\hline \multirow{2}{*}{ Elit } & & 0 & 100.0 \\
\hline
\end{tabular}

a. Eliminación por lista basada en todas las variables del procedimiento.

Fuente: Elaboración propia

Tabla 5. Alfa de Cronbach para instrumento de profesores

\begin{tabular}{|c|c|c|}
\hline & \multicolumn{2}{|c|}{ Estadísticos de fiabilidad } \\
\hline $\begin{array}{c}\text { Alfa de } \\
\text { Cronbach }\end{array}$ & $\begin{array}{c}\text { Alfa de Cronbach basada } \\
\text { en elementos } \\
\text { estandarizados }\end{array}$ & N de elementos \\
\hline .810 & .814 & 15 \\
\hline
\end{tabular}

Fuente: Elaboración propia

Analizados los resultados, se puede decir que se obtuvo un instrumento con consistencia buena; además, es válido y confiable. Finalmente, fue aplicado de manera digital (mediante Google Forms) a todos los profesores que cursaron y aprobaron el curso (116).

En cuanto a las generalidades de los resultados, $60 \%$ de los encuestados eran hombres y $40 \%$ mujeres; la edad mínima fue de 25 años y la máxima de 65 años (edad promedio = 45 años). En promedio, los encuestados tienen casi 15 años de impartir clases. Asimismo, $59 \%$ cuentan con doctorado, $30 \%$ maestría, $1 \%$ especialización y $8 \%$ licenciatura.

Los resultados conseguidos en cuanto a la dimensión de capacitación reflejan que $99 \%$ están muy de acuerdo y de acuerdo con que el curso fue de utilidad para mejorar la impartición de sus clases en línea. Esto evidencia la importancia de una correcta capacitación (figura 5). 

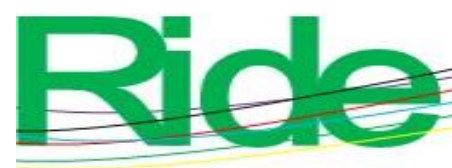

Revista Iberoamericana para la
nvestigación y el Desarrollo Educativo

ISSN 2007-7467

Figura 5. Utilidad del curso-taller para mejorar de la impartición de clases

Considera que el curso fue de utilidad para mejorar la impartición de su clase como facilitador en línea

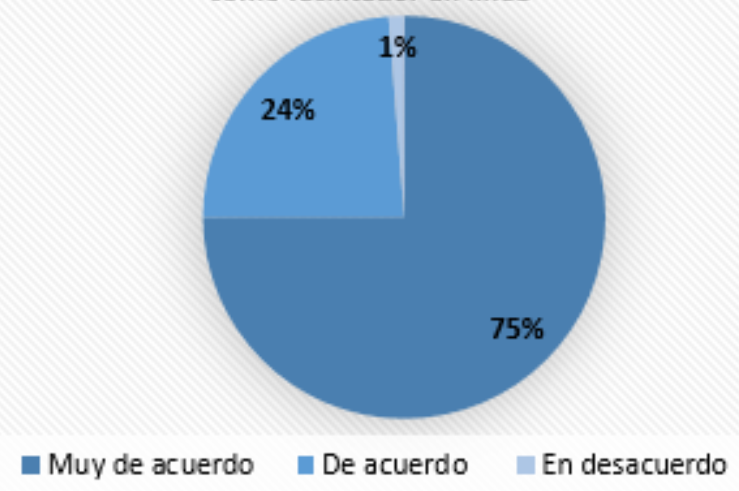

Fuente: Elaboración propia

Asimismo, $97 \%$ de los encuestados respondieron que están muy de acuerdo y de acuerdo con la metodología del diseño instruccional, pues mejoraron su planeación didáctica en beneficio de los procesos de enseñanza-aprendizaje (figura 6).

Figura 6. Utilidad del curso-taller para mejorar de la impartición de clases

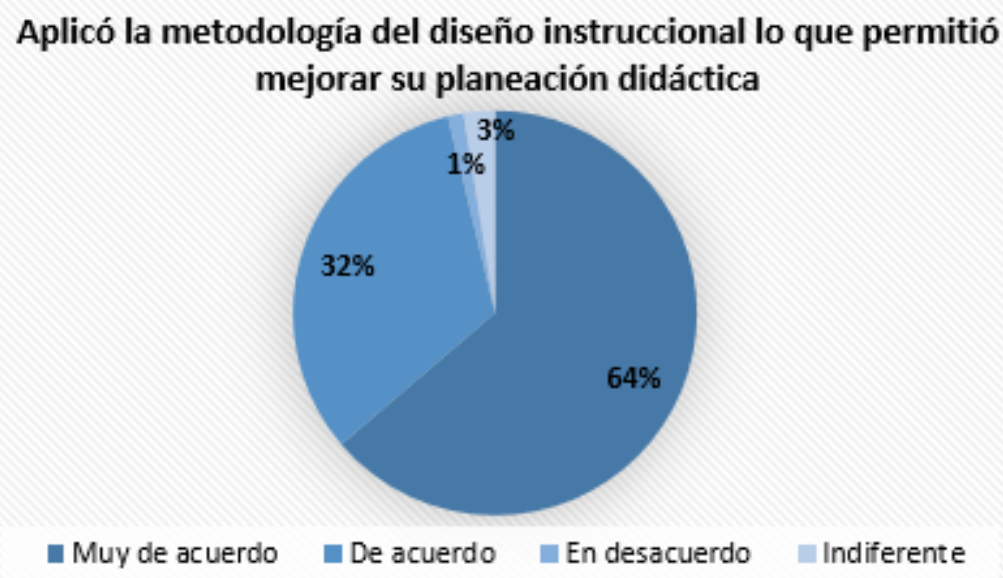

Fuente: Elaboración propia

En lo referente a la dimensión de tecnología, 87 \% de los profesores usó al menos una aplicación para diseñar y editar audios y videos, aspecto elemental para crear recursos educativos digitales que favorezcan el aprendizaje de los estudiantes (figura 7). 

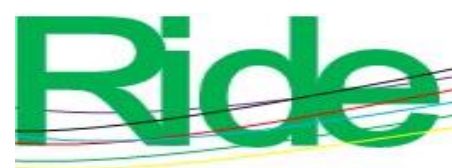

Figura 7. Uso de recursos tecnológicos por parte de los profesores

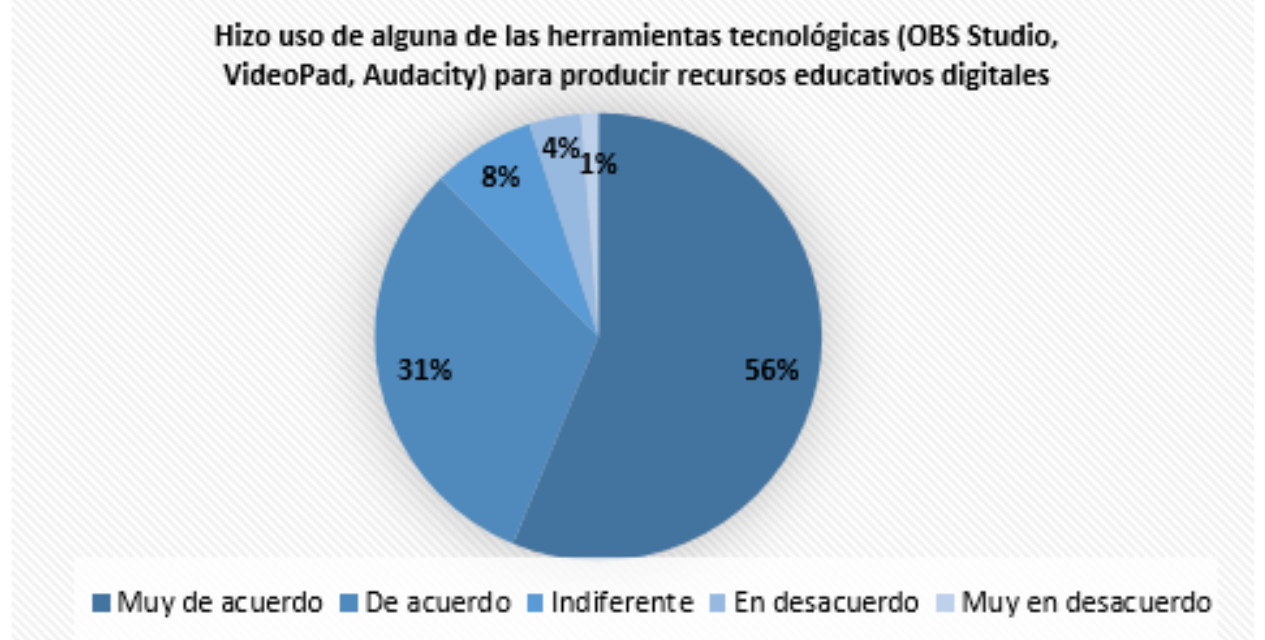

Fuente: Elaboración propia

Además de los recursos tecnológicos propuestos en el curso, $63 \%$ utilizó la plataforma institucional de Eminus (en su versión 3 o 4) para cargar los contenidos didácticos, diseñar actividades de aprendizaje y evaluar el curso. En cuanto a herramientas para actividades síncronas, $56 \%$ empleó la plataforma de Microsoft Teams, y $44 \%$ otras como Zoom y Google Meet.

En lo referente a la dimensión de aprendizaje, $94 \%$ consideró que aplicando la metodología propuesta para el diseño instruccional y empleando los recursos educativos digitales en la modalidad mixta, hubo mejoras en el aprendizaje de los estudiantes, lo que demuestra que este tipo de proyectos resultan muy útiles (figura 8). 

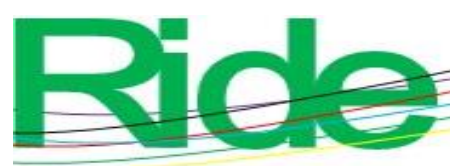

Revista Iberoamericana para la
yestigación el Desarrollo Educativo

Figura 8. Resultado del proceso de aprendizaje de los estudiantes

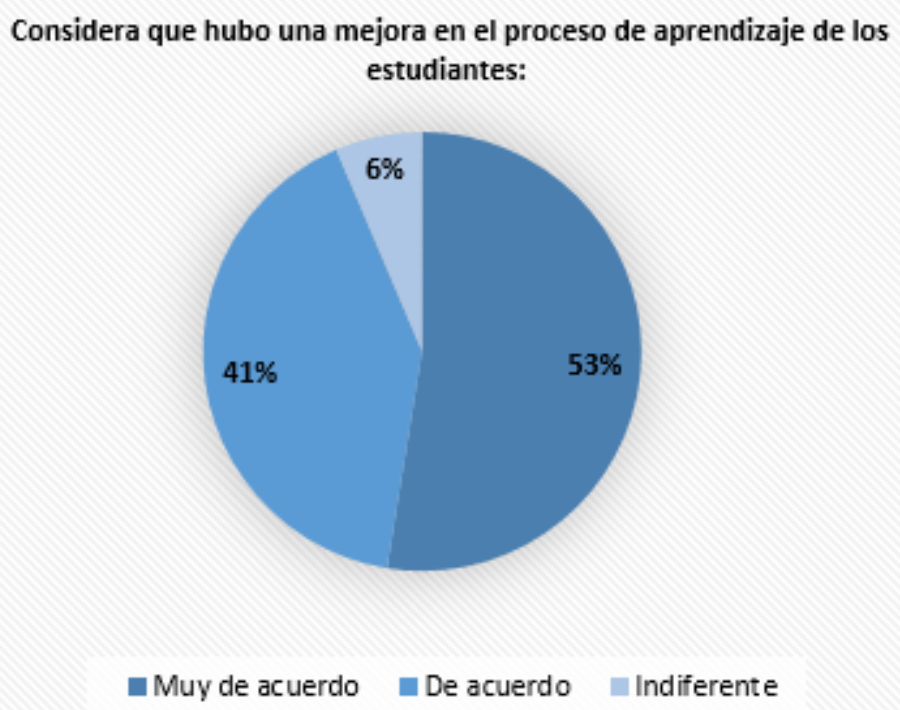

Fuente: Elaboración propia

En cuanto a la participación de los estudiantes en los cursos en línea, $92 \%$ de los profesores respondieron que están muy de acuerdo y de acuerdo con que hubo una mejora en la entrega de sus actividades (figura 9).

Figura 9. Uso de aplicaciones tecnológicas

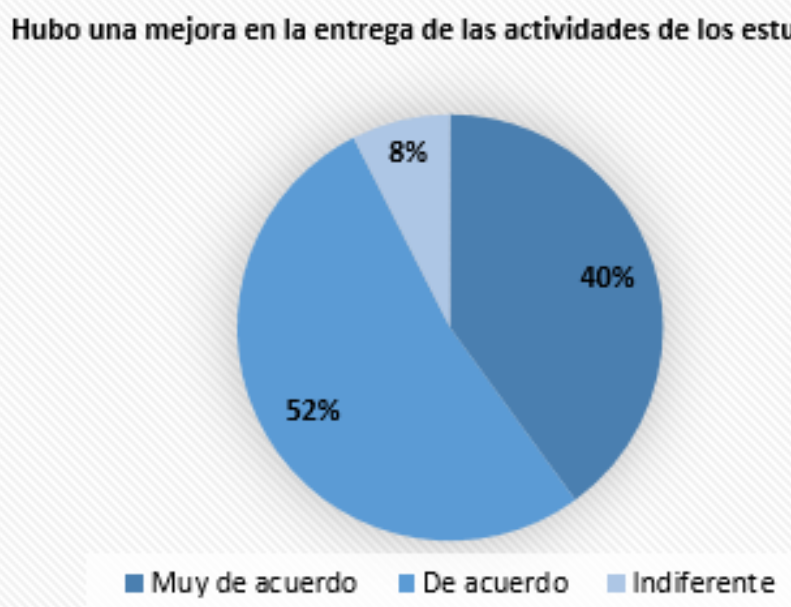

Fuente: Elaboración propia

En la figura 10 se observa que $80 \%$ de los profesores respondieron que el curso-taller sirvió para que la acreditación de sus estudiantes mejorara. 


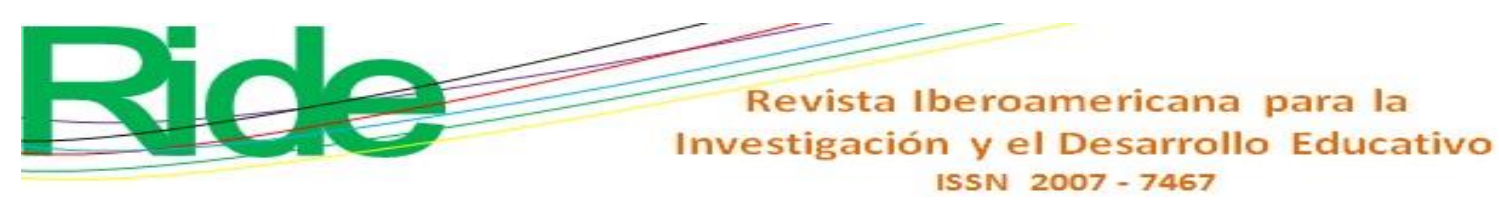

Figura 10. Mejora en la acreditación del curso

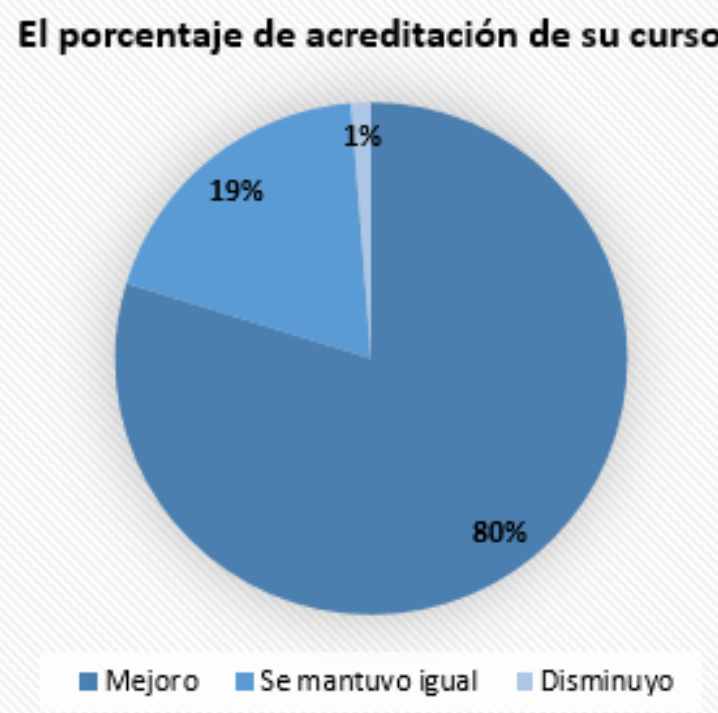

Fuente: Elaboración propia

\section{Análisis correlacionales de datos}

Además de los resultados descriptivos de la investigación, se llevó a cabo un análisis correlacional de datos por medio de la estadística inferencial no paramétrica, empleando la metodología de Tau-b de Kendall para tablas cuadradas y el Tau-c de Kendall para no cuadradas.

En primer término, se analizó si existió alguna asociación entre la aplicación de la metodología para el diseño instruccional de cursos en línea y la mejora en el proceso de aprendizaje de los estudiantes.

Las hipótesis formuladas fueron las siguientes:

- $\mathrm{H}_{0}$ : No existe relación significativa entre la aplicación de la metodología para el diseño instruccional y la mejora en el proceso de aprendizaje de los estudiantes.

- $\mathrm{H}_{1}$ : Existe relación significativa entre la aplicación de la metodología para el diseño instruccional y la mejora en el proceso de aprendizaje de los estudiantes.

Si la sig. p-valor es $<0.05$ se rechaza $\mathrm{H}_{0}$; en caso contrario se acepta. Método Tau-b de Kendall. 


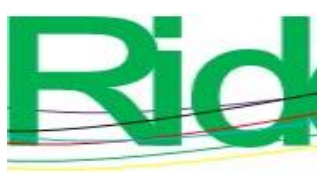

Revista Iberoamericana para la Investigación y el Desarrollo Educativo

ISSN 2007 - 7467

Tabla 6. Correlación diseño instruccional-proceso de aprendizaje de estudiantes

\begin{tabular}{|l|l|l|c|c|}
\hline \multicolumn{5}{|c|}{ Correlaciones } \\
\hline \multirow{4}{*}{$\begin{array}{l}\text { Tau_b } \\
\text { de } \\
\text { Kendall }\end{array}$} & \multirow{3}{*}{$\begin{array}{l}\text { Metodología } \\
\text { Kenay }\end{array}$} & $\begin{array}{l}\text { Coeficiente de } \\
\text { correlación }\end{array}$ & 1.000 & .435 \\
\cline { 3 - 5 } & & Sig. (bilateral) &. & .152 \\
\cline { 3 - 5 } & Aprendizaje & $\begin{array}{l}\text { Coeficiente de } \\
\text { correlación }\end{array}$ & .435 & 116 \\
\cline { 3 - 5 } & & Sig. (bilateral) & .000 & 1.000 \\
\cline { 3 - 5 } & $\mathrm{N}$ & 116 & 116 \\
\hline
\end{tabular}

Fuente: Elaboración propia

Como se puede apreciar en la tabla 6, según el p-valor "Sig. (bilateral)" $=.000<.05$, lo que lleva a rechazar la $\mathrm{H}_{0}$. Se concluye, por tanto, que hay una asociación altamente significativa entre las variables de la aplicación de metodología del diseño instruccional por parte de los profesores y la mejora en el proceso de aprendizaje de los estudiantes. Esto queda evidenciado con el valor de coeficiente de correlación $\mathrm{t}=0.435$, el cual se interpreta como una correlación positiva moderada entre las variables.

Posteriormente se analizó si existió alguna asociación entre el diseño de recursos educativos digitales por parte de los profesores y la mejora en la acreditación de la materia por parte de los estudiantes.

Las hipótesis fueron las siguientes:

- $\mathrm{H}_{0}$ : No Existe relación significativa entre la implementación de recursos educativos digitales y la mejora en la acreditación de las materias por parte de estudiantes.

- $\mathrm{H}_{1}$ : Existe relación significativa entre la implementación de recursos educativos digitales y la mejora en la acreditación de las materias por parte de estudiantes $\mathrm{Si}$ la sig. p-valor es < 0.05 se rechaza $\mathrm{H}_{0}$; en caso contrario se acepta. Método Tau-c de Kendall.

Tabla 7. Correlación recursos educativos digitales-aprobación de materias

\begin{tabular}{|l|r|r|r|r|r|}
\hline \multicolumn{2}{|c|}{ Medidas simétricas } \\
\hline \multicolumn{2}{|c|}{} & $\begin{array}{c}\text { Valo } \\
\mathrm{r}\end{array}$ & $\begin{array}{c}\text { Error } \\
\text { estándar } \\
\text { asintótico }\end{array}$ & $\begin{array}{c}\text { T } \\
\text { aproximada } \\
\text { b }\end{array}$ & $\begin{array}{c}\text { Significaci } \\
\text { ón } \\
\text { aproximada }\end{array}$ \\
\hline $\begin{array}{l}\text { Ordinal por } \\
\text { ordinal }\end{array}$ & $\begin{array}{l}\text { Tau-c de } \\
\text { Kendall }\end{array}$ & .155 & .070 & 2.213 & .027 \\
\hline \multicolumn{2}{|l|}{ N. ${ }^{\circ}$ de casos válidos } & 116 & & & \\
\hline
\end{tabular}

Fuente: Elaboración propia 


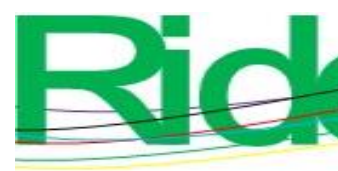

Revista Iberoamericana para la Investigación y el Desarrollo Educativo ISSN 2007-7467

En tal sentido, los resultados de esta investigación enseñan que ocho de cada diez profesores que participaron en el proyecto consideran que existió un incremento en la acreditación de la materia por parte de los estudiantes, dato importante que permite reducir los porcentajes de reprobación y de deserción. Los resultados descriptivos están alineados con los inferenciales, ya que demostraron que existe una relación entre la implementación de recursos educativos digitales y la mejora en la acreditación de las materias por parte de los estudiantes.

Por tal motivo, podemos concluir que esta iniciativa cumplió con el objetivo de capacitar a los docentes en el uso de herramientas digitales, conocimiento indispensable para desenvolverse con éxito en entornos virtuales de enseñanza y aprendizaje. Por eso, este tipo de capacitación debe ampliarse a otras áreas académicas. En síntesis, esta investigación tiene una importancia fundamental, pues se relaciona con los nuevos modelos de aprendizaje.

\section{Futuras líneas de investigación}

La continuidad de esta investigación en futuras líneas debe enfocarse en dos aspectos primordiales: primero, seguir con la capacitación docente, ya que los resultados reflejan que $98 \%$ de los profesores considera que el diseño instruccional y la elaboración de recursos educativos digitales sirve para mejorar sus prácticas didácticas. Segundo, se debe indagar en la percepción de los estudiantes con respecto a la mejora de las competencias de los docentes para la impartición de clases virtuales, pues de nada sirve evaluar el proceso de enseñanza si no se tiene conocimiento de su impacto en el proceso de aprendizaje. Por ello, se está diseñando un instrumento para recabar datos cuantitativos y cualitativos sobre el aprendizaje de los estudiantes, el cual debe ser aplicado a un grupo de control para identificar áreas de oportunidad en la mejora del proyecto. 


\section{Referencias}

Aguaded, J. I. y Muñoz, M. (2012). La competencia digital en el alumnado con necesidades especiales. Uso de ordenadores, tabletas, internet, e-books y narraciones digitales en el aula (pp. 1254-1261). En I Congreso Virtual Internacional sobre Innovación Pedagógica y Praxis Educativa INNOVAGOGIA 2012. Sevilla. Recuperado de http://rabida.uhu.es/dspace/bitstream/handle/10272/7505/La_competencia_digital_a lumnado.pdf?sequence $=2$

Andrade, E. y Velázquez, E. (2014). La biblioteca de la Facultad de Ingeniería en la plataforma educativa de la UdelaR - Uruguay: implementando un servicio de formación de usuarios virtual. Revista Interamericana de Bibliotecología, 37(2), 171178.

Belloch, C. (2013). Diseño instruccional. España: Unidad de Tecnología Educativa. Universidad de Valencia. http://www.uv.es/ bellochc/pedagogia/EVA4.pdf

Brambila, L., Camarena Cadena, M., Orozco Valerio, M. y Vizcaíno, A. (2006). El diseño instruccional como proceso coadyuvante de identidad profesional del trabajador social. Apertura, 6(4), 31-43. $\quad$ Recuperado de https://www.redalyc.org/articulo.oa?id=688/68800404

Branch, R. and Kopcha, T. J. (2014). Instructional design models. In Michael Spector, J., David Merrill, M., Jan, E. and Bishop, M. J. (eds.), Handbook of Research on Educational Communications and Technology (pp. 77-87). Nueva York: Springer. Doi: https://doi.org/10.1007/978-1-4614-3185-5_7, 10.1007/978-1-4614-3185-5_7

Candia, F. (2016). Diseño de un modelo curricular E-learning, utilizando una metodología activa participativa. RIDE. Revista Iberoamericana para la Investigación y el Desarrollo Educativo, 7(13), 147-182. Recuperado de http://www.scielo.org.mx/scielo.php?script=sci_arttext\&pid=S200774672016000200147\&lng=es\&tlng=es

Comunidades Digitales para el Aprendizaje en Educación Superior (CODAES) (2015). Modelo de diseño instruccional de la CODAES.

Colunga, A. (2005) Eminus. Sistema de Educación distribuida (diapositivas). Dirección General de Tecnología de Información. Universidad Veracruzana. Recuperado de http://www.cudi.edu.mx/primavera_2005/presentaciones/alejandro_colunga.pdf 


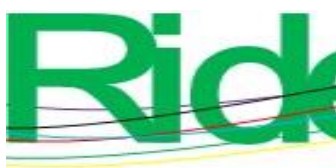

Revista Iberoamericana para la Investigación y el Desarrollo Educativo ISSN 2007 - 7467

Domínguez, C., Organista Sandoval, J. y López Ornelas, M. (2018). Diseño instruccional para el desarrollo de contenidos educativos digitales para teléfonos inteligentes. Apertura, $\quad$ 10(2), 80-93. Recuperado de https://www.redalyc.org/articulo.oa?id=688/68857368006

Gazca, L. A. (2020). Implicaciones del coronavirus covid-19 en los procesos de enseñanza en la educación superior. RIDE Revista Iberoamericana Para La Investigación y el Desarrollo Educativo, 11(21). Doi: https://doi.org/10.23913/ride.v11i21.753

George, D. y Mallery, P. (2003). SPSS for Windows step by step: A Simple Guide and Reference. 11.0 Update (4. ${ }^{\mathrm{a}}$ ed.). Boston: Allyn \& Bacon.

López, M. y Chávez Espinoza, J. (2013). La formación de profesores universitarios en la aplicación de las TIC. Sinéctica, (41), 2-18. Recuperado de http://www.scielo.org.mx/scielo.php?script=sci_arttext\&pid=S1665109X2013000200005\&lng=es\&tlng=es

Luna, M., Ayala S. y Rosas, P., et al. (2021). Diseño instruccional empático. Un modelo de encuentro entre personas. Astra Ediciones. Recuperado de https://www.researchgate.net/publication/351637590_Diseno_Instruccional_Empati co_Un_modelo_de_encuentro_entre_personas

Margalef, I. y García, C. (2016). La aplicación de un recurso educativo digital en la dificultad de aprendizaje de la resta: un estudio de caso. Revista Electrónica Educare, 20(1), 122. Recuperado de https://www.redalyc.org/articulo.oa?id=1941/194143011013

Moreno, M., Contreras Domínguez, I., Gómez Jiménez, S. y Martínez Velázquez, L. (2014). Análisis de un diseño instruccional para aplicarlo en unidades curriculares híbridas. Revista Iberoamericana de Producción Académica y Gestión Educativa, 1(1). Recuperado de http://www.pag.org.mx/index.php/PAG/article/view/121/168.w

Raichman, S. y Mirasso, A. (2018). Modelos pedagógicos para el aprendizaje complejo y la formación en competencias en carreras de Ingeniería. Ingeniería, 22(3). Recuperado de https://www.redalyc.org/articulo.oa?id=467/46759491008

Unesco (2004). Las tecnologías de la información y la comunicación en la formación docente. Guía de planificación. Ediciones TRILCE.

Universidad Veracruzana (2020). Disposiciones Generales para el Trabajo Académico. Recuperado de https://www.uv.mx/veracruz/adtusi/files/2020/07/DispGen17julio.pdf 


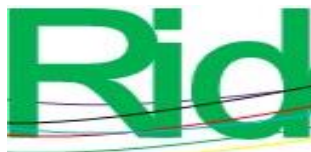

Universidad Veracruzana

(2021).

EMINUS.

Recuperado

de https://www.uv.mx/plataformasacademicas/eminus

Zapata, M. (2012). Recursos educativos digitales: conceptos básicos. Aprende en Línea. Recuperado de https://aprendeenlinea.udea.edu.co/boa/contenidos.php/d211b52ee1441a30b59ae00 8e2d31386/845/estilo/aHR0cDovL2FwcmVuZGVlbmxpbmVhLnVkZWEuZWR1L mNvL2VzdGlsb3MvYXp1bF9jb3Jwb3JhdG12by5jc3M=/1/contenido/\#referencia_3 a 


\section{ANEXO 1}

Figura 10. Instrumento de evaluación del proyecto de intervención para la capacitación virtual de profesores de educación superior en diseño instruccional y recursos educativos digitales.

Estimado profesor:

El presente instrumento tiene como objetivo recabar información que nos permita identificar la percepción actual de los académicos que tomaron el curso sobre Diseño instruccional y recursos educativos digitales referente al impacto que turo en la impartición cle sus clases en linea en el periodo agosto 2020-febrero 2021, por lo que solicitamos su valioso apoyo para contestar todos los items de manera objetiva y siguiendo las instrucciones. Le agradecemos de antemano su interés y decidido apoyo al responder este cuestionario.

GENERALIDADES

\begin{tabular}{|l|c|c|c|c|c|}
\hline Marque con una X su sexo & M & F & & Escriba su edad & \\
\hline $\begin{array}{l}\text { Marque con una X } \\
\text { su último grado de } \\
\text { estudios }\end{array}$ & Licenciatura & Especialidad & Maestria & Doctorado \\
\hline $\begin{array}{l}\text { Marque con una X su tipo de } \\
\text { contratación }\end{array}$ & $\begin{array}{l}\text { Profesor de tiempo } \\
\text { completo }\end{array}$ & $\begin{array}{c}\text { Profesor investigador } \\
\text { de tiempo completo }\end{array}$ & Profesor de asiguatura \\
\hline \begin{tabular}{l} 
Especifique el número de años impartiendo clases \\
\hline
\end{tabular}
\end{tabular}

\section{INSTRUCCIONES}

Marque con una X la casilla que considere correspondiente al grado de identificación en cada ítem tomando en cuenta las siguientes categorias:

\begin{tabular}{|c|c|c|c|c|c|c|c|c|}
\hline 1 & 2 & 3 & 4 & \multicolumn{5}{|c|}{5} \\
\hline Totalmente en desacuerdo & En desacuerdo & Indiferente & De acuerdo & & Total & lent & de & aerdo \\
\hline \multicolumn{4}{|c|}{ Items } & $(-) 1$ & 2 & 3 & 4 & $5(+)$ \\
\hline \multicolumn{9}{|c|}{ Dimensión capacitación } \\
\hline \multicolumn{4}{|c|}{$\begin{array}{l}\text { 1. Previo al curso, habia tomado alguna capacitación en el ámbito de la tecnología } \\
\text { educativa }\end{array}$} & & & & & \\
\hline \multicolumn{4}{|c|}{$\begin{array}{l}\text { 2. Considera que el curso fue de utilidad para mejorar la impartición de su clase } \\
\text { como facilitador en límea }\end{array}$} & & & & & \\
\hline \multicolumn{4}{|c|}{$\begin{array}{l}\text { 3. Pudo aplicar la metodología del diseño instruccional, lo que permitió mejorar } \\
\text { su planeación didáctica }\end{array}$} & & & & & \\
\hline \multicolumn{4}{|c|}{$\begin{array}{l}\text { 4. Considera adecuada la metodologia de diseño instruccional propuesta en el } \\
\text { curso }\end{array}$} & & & & & \\
\hline \multicolumn{9}{|c|}{ Dimensión tecnología } \\
\hline \multicolumn{9}{|c|}{ 5. Considera adecuadas los recursos tecnológicos propuestos en el curso } \\
\hline \multicolumn{9}{|c|}{ 6. Hizo uso del recurso tecnológico OBS Studio para el diseño de videos } \\
\hline \multicolumn{9}{|c|}{ 7. Hizo uso del recurso tecnológico VideoPad para la edición de videos } \\
\hline \multicolumn{9}{|c|}{ 8. Hizo uso del recurso tecnológico Audacity para el diseño de audios } \\
\hline \multicolumn{9}{|c|}{ 9. Considera que bubo una mejora en el proceso de aprendizaje de los estudiantes } \\
\hline \multicolumn{9}{|c|}{$\begin{array}{l}\text { 10. Además de los recursos tecnológicos que aprendió en el curso, mencione algunos otros que utilizó en la } \\
\text { impartición de su clase (puede elegir más de una opción): } \\
\text { Eminus }\end{array}$} \\
\hline \multicolumn{9}{|c|}{ Dimensión aprendizaje } \\
\hline \multicolumn{9}{|c|}{ 11. Hubo una mejora en la entrega de las actividades de los estudiantes } \\
\hline \multicolumn{9}{|c|}{ 12. Mejoró la calidad de los trabajos o proyectos entregados por los estudiantes } \\
\hline \multicolumn{9}{|c|}{$\begin{array}{l}\text { 13. Hubo comentarios favorables de parte de los estudiantes sobre la forma en que } \\
\text { impartió el curso }\end{array}$} \\
\hline \multicolumn{5}{|c|}{ 14. El porcentaje de acreditación de su curso: } & & & & \\
\hline $\begin{array}{l}\text { 15. Considera que se debe da } \\
\text { el uso de metodologias } \mathrm{C}\end{array}$ & $\begin{array}{l}\text { tinuidad a este } \\
\text { uso de recurso: }\end{array}$ & $\begin{array}{l}\text { le capacitació } \\
\text { ológicos edu }\end{array}$ & ra mejorar & & & & & \\
\hline
\end{tabular}

Fuente: Elaboración propia 